\title{
Bioanalysis
}

\section{Implementing fit-for-purpose biomarker assay approaches: a bioanalytical perspective}

\author{
"For biomarker assays, distinct experiments and approaches for \\ biomarker assays need to be considered in comparison to PK assays."
}

First draft submitted: 14 March 2016; Accepted for publication: 4 April 2016;

Published online: 6 May 2016

Keywords: assay $\bullet$ biomarkers $\bullet$ calibrator $\bullet$ fit-for-purpose $\bullet$ parallelism $\bullet$ validation

\section{Fit-for-purpose biomarker assay approaches}

Use of a successful biomarker strategy can facilitate drug development in several ways. Biomarkers can aid in the demonstration of drug target engagement, help to determine the appropriate drug dosage, provide early assessment of drug safety and, potentially, assist in optimizing efficacy via patient selection. To do so, biomarker assays with acceptable analytical performance are essential for producing reliable results. The fit-for-purpose (FFP) approach for biomarker assays, which has been described by many recent publications [1-10], should ensure that the analytical performance of the assay meets the minimal acceptance criteria to support the intended purpose of the data. The most important challenge is to ensure that the analyte quantitated by the assay is in fact the biomarker of interest, the primary challenge being that the calibrator material usually does not match the endogenous analyte. As another consideration per the FFP approach, the methods should support efficient and therefore cost-effective decision-making in the drug-development process.

Publication of the 2013 FDA Draft Guidance for Bioanalytical Method Validation [11] was the first guidance to formally discuss the FFP approach in the context of bioanalytical methods for biomarkers. The draft guidance emphasized that the extent to which a method is validated is a decision left to the sponsor, unless the biomarker data are intended to support label claims. The FFP approach has been employed by a few subsequent publications [12-14]. Nonetheless, it is still being discussed across the industry, in particular to seek clarity with regard to when a sponsor can elect to not validate an assay. Very recently, in September 2015, the Crystal City VI Workshop assembled bioanalytical experts from across the industry, including scientists from the US FDA [15]. The group addressed the need for clarity on many specific questions related to biomarker assay characterization. Follow-up discussions are planned at several meetings, including at the American Association of Pharmaceutical Scientists National Biotech Conference, at the Workshop on Recent Issues in Bioanalysis, and will continue to occur at several forums internationally. Many key points that need clarification or require consensus continue to engage many scientists across the industry. This is a brief summary of my current opinions on implementing the FFP approach for biomarker assays, understanding that every scientist should approach each biomarker assay with the appropriate scientific rigor.

\section{Validation versus qualification}

For each bioanalytical strategy, the FFP approach should be implemented, making sure that a given biomarker assay is characterized to the extent that is suitable for its intended use. Biomarker data that impact safety in either nonclinical or clinical studies should be derived from fully validated assays.

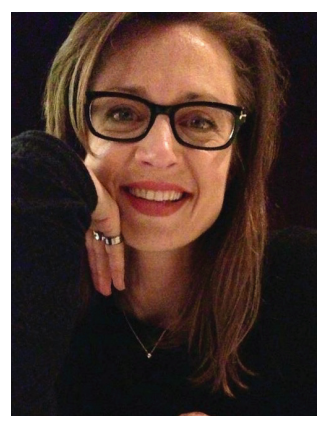

Kyra J Cowan Genentech, Inc., BioAnalytical Sciences, 1 DNA Way, South San Francisco, CA 94080, USA

Tel.: +1 6504675053

Fax: +16502251998

cowan.kyra@gene.com 
Biomarker assays that have been selected to support pivotal Phase III studies, the results of which may be included in drug labeling, should also be fully validated. For exploratory biomarkers, which tend to be more common in nonclinical studies and during Phase I and II clinical trials, our laboratories use an approach called qualification. In these cases, we implement $a$ priori acceptance criteria for the assay parameters tested, but do not reference standard operating procedures. The qualification procedure requires significantly fewer reagents and assay runs; it also examines fewer assay parameters in comparison to validation, including the evaluations for stability, robustness and ruggedness. This saves significantly in time and resources, while maintaining the appropriate scientific rigor. The experiments that we perform show that each assay is 'relative quantitative' as described recently in the Crystal City V Workshop white paper [16], based on the precision of our parallelism data and the performance of the assay calibrators and controls. For a validated assay, we perform additional experiments that include extensive stability tests, robustness and ruggedness and lot-to-lot variability. It is important to note here that almost all well characterized biomarker assays, whether qualified or validated, are relative quantitative. In rare instances, an assay has been defined as 'definitive quantitative' [16], when the characterization of a biomarker assay included an assessment of a WHO reference standard that is known to be equivalent to the endogenous protein. However, unlike PK assays, the endogenous analyte could still be somewhat different than such an extensively characterized recombinant material, which must be taken into consideration.

\section{"Experience has shown that validation of a biomarker assay is not always needed and that a clearly defined fit-for-purpose approach saves resources and can accelerate data delivery to our molecule portfolio teams."}

\section{Key assay parameters}

It is important to note that there are key differences between biomarker assays and PK assays. The 2013 FDA draft guidance [11] recommends that method validation for biomarker assays address the same questions as that of PK assays. For biomarker assays, distinct experiments and approaches for biomarker assays need to be considered in comparison to PK assays. The key difference between PK and biomarker assays is the calibrator material. PK assays are developed with reference material that is equivalent to what is used in the corresponding nonclinical or clinical studies. However, for most biomarker assays, recombinant material that may not be truly equivalent to the endogenous target is used. A key challenge is ensuring that the recombinant material used as the calibrator material, and oftentimes used for quality controls, is representative of the endogenous analyte of interest. Scientists should understand the biology of the endogenous biomarker including, but not limited to, post-translational modifications, multimerization, the presence and impact of binding proteins in matrices as well as the anticipated endogenous levels of the biomarker, both in normal healthy volunteers or animals as well as in the disease-state patient population matrices, where possible. Choosing a surrogate matrix to be used in the biomarker assay calibration curve is also important if the target matrix cannot be used due to endogenous analyte levels.

Some assay parameters that are assessed for PK assays generally do not apply to biomarker assays, although similar concepts hold. Importantly, accuracy is not a shared assay parameter. Accuracy in biomarker assays is challenging in comparison to $\mathrm{PK}$ assays because the recombinant material may not be representative of the endogenous analyte. For that reason, we generally term accuracy for biomarker assays as 'relative accuracy.' For the same reason, stability is challenging to confirm because the recombinant material may behave differently compared with the endogenous analyte under varying conditions. Most importantly, and regardless of the level of characterization for an FFP approach, parallelism is a key experiment that must be conducted early in the evaluation of the assay (if there are sufficient endogenous levels) to ensure that the recombinant material prepared in a surrogate matrix has the same concentration-response relationship as the samples to be tested. This experiment differs from dilutional linearity, in which case a reference material is spiked into a matrix and diluted serially. The parallelism experiment will also confirm the minimum required dilution and selectivity of the assay, the appropriateness of the surrogate matrix in the calibrator curve (and controls if used) and the detectability of the analyte in the target matrix.

\section{Conclusion}

Experience has shown that validation of a biomarker assay is not always needed and that a clearly defined FFP approach saves resources and can accelerate data delivery to our molecule portfolio teams. If the biomarker results are intended to support label claims, the FFP strategy must include validation of the biomarker assay. The selection and rigor of experiments for a biomarker assay are dependent on the ultimate purpose for which the data are intended. Bioanalytical scientists should communicate early with drug-development 
teams on the caveats associated with biomarker assay qualification versus validation. In this way, the development team will understand the risks associated with assays that are less characterized than those that are fully validated and documented. This is especially important if there are unexpected results from sample analysis. This approach ensures that there is a broader understanding around the tiered approach for characterizing a biomarker assay.

\section{References}

1 Desilva B, Smith W, Weiner R et al. Recommendations for the bioanalytical method validation of ligand-binding assays to support pharmacokinetic assessments of macromolecules. Pharm. Res. 20(11), 1885-1900 (2003).

2 Lee JW, Weiner RS, Sailstad JM et al. Method validation and measurement of biomarkers in nonclinical and clinical samples in drug development: a conference report. Pharm. Res. 22(4), 499-511 (2005).

3 Lee JW, Devanarayan V, Barrett YC et al. Fit-for-purpose method development and validation for successful biomarker measurement. Pharm. Res. 23(2), 312-328 (2006).

4 Chau CH, Rixe O, Mcleod H, Figg WD. Validation of analytic methods for biomarkers used in drug development. Clin. Cancer Res. 14(19), 5967-5976 (2008).

5 Lee JW, Hall M. Method validation of protein biomarkers in support of drug development or clinical diagnosis/prognosis. J. Chromatogr. B Analyt. Technol. Biomed. Life Sci. 877(13), 1259-1271 (2009).

6 Cummings J, Raynaud F, Jones L, Sugar R, Dive C. Fitfor-purpose biomarker method validation for application in clinical trials of anticancer drugs. Br. J. Cancer 103(9), 1313-1317 (2010).

7 Cummings J, Ward TH, Dive C. Fit-for-purpose biomarker method validation in anticancer drug development. Drug Discov. Today 15(19), 816-825 (2010).

8 Brookes K, Cummings J, Backen A et al. Issues on fit-forpurpose validation of a panel of ELISAs for application as biomarkers in clinical trials of anti-angiogenic drugs. $\mathrm{Br}$. J. Cancer 102(10), 1524-1532 (2010).

\section{Financial \& competing interests disclosure}

The author is an employee of Genentech, Inc. and a stockholder of the Roche Group. The author has no other relevant affiliations or financial involvement with any organization or entity with a financial interest in or financial conflict with the subject matter or materials discussed in the manuscript apart from those disclosed.

No writing assistance was utilized in the production of this manuscript.

9 Khan MU, Bowsher RR, Cameron M et al. Recommendations for adaptation and validation of commercial kits for biomarker quantification in drug development. Bioanalysis 7(2), 229-242 (2015).

10 Jani D, Allinson J, Berisha F et al. Recommendations for use and fit-for-purpose validation of biomarker multiplex ligand binding assays in drug development. AAPS J. 18(1), 1-14 (2016).

11 FDA. Draft Guidance for Industry. Bioanalytical method validation (2013). www.fda.gov

12 Lin M, Lassman ME, Weiner R, Laterza OF. Development and fit-for-purpose validation of a LC-MS/MS assay for fibrinogen peptide a quantitation in human plasma. Bioanalysis 6(13), 1759-1766 (2014).

13 Ni YG, Yuan X, Newitt JA et al. Development and fit-forpurpose validation of a soluble human programmed death-1 protein assay. AAPS J. 17(4), 976-987 (2015).

14 Gregg EO, Minet E, McEwan M. Urinary biomarkers of smokers' exposure to tobacco smoke constituents in tobacco products assessment: a fit for purpose approach. Biomarkers 18(6), 467-486 (2013).

15 Lowes S, Ackermann B. AAPS and US FDA Crystal City VI workshop on bioanalytical method validation for biomarkers. Bioanalysis 8(3), 163-167 (2016).

16 Booth B, Arnold ME, DeSilva B et al. Workshop report: Crystal City V-quantitative bioanalytical method validation and implementation: The 2013 Revised FDA Guidance. AAPS J. 17(2), 277-288 (2015). 